\title{
Review of: "Molecular insights into the interaction of HPV-16 E6 variants against MAGI-1 PDZ1 domain"
}

\author{
Ana Elvira Zacapala Gómez, Marco Antonio Leyva-Vazquez ${ }^{1}$
}

1 Universidad Autónoma de Guerrero

Potential competing interests: The author(s) declared that no potential competing interests exist.

It is a very interesting in silico article because the results show that E6 of the HPV16 variants have a higher affinity for MAGI-1 compared to E6-Reference. Considering that MAGI-1 is a protein involved in cell junctions and that E6 binds MAGI-1, these results suggest that E6 of HPV16 variants may be more oncogenic compared to E6 of reference.

The authors present a clear redaction to describe the methodology used and describe the results, which facilitates their understanding. 